Pacific Journal of Mathematics

INTEGRAL EQUIVALENCE OF VECTORS OVER LOCAL 


\title{
INTEGRAL EQUIVALENCE OF VECTORS OVER LOCAL MODULAR LATTICES
}

\author{
JoHN S. HsIA
}

Let $F$ be a local field with characteristic unequal to two, and in which the element 2 is not unitary. Let $V$ be a regular quadratic space over $F, L$ a lattice on $V$. The group of units of $L$ is the subgroup

$$
0(L)=\{\sigma \in 0(V) \mid \sigma L=L\}
$$

of the orthogonal group $0(V)$. Two vectors $u$ and $v$ in $L$ are defined to be integrally equivalent if there exists an isometry $\sigma \in 0(L)$ mapping one onto the other. This paper gives necessary and sufficient conditions for integral equivalence of vectors when the underlying lattice $L$ is modular.

A very fundamental theorem in all studies of quadratic forms is the well-known Witt's Theorem. Yet, integral versions of it come scarce. However, there has been some stirring signs of interest and activity of late along this direction. The solution for integral equivalence of vectors would, of course, constitute an one-dimensional integral extension of this classic theorem. Recent works by James [3], Knebusch [4], Rosenzweig [8], Trojan [9], and Wall [10] may be consulted for the few known special cases. Earlier in [2] the author had extended Trojan's unramified modular solution to the special case of the so-called depleted modular lattices over any dyadic local field. This paper removes the restriction to the size of the weight ideal associated with the lattice and thereby completes the solution for arbitrary modular lattices over dyadic local fields.

The technicalities involved when dealing with an arbitrary lattice are substantial and not all of which we have been able to overcome. Here again special cases have been solved and they are included in the author's doctoral dissertation [1].

1. Preliminaries. We shall freely make use of the results and terminologies of [6]. We do, however, wish to emphasize a few important relevant facts.

The ground field $F$ is a fixed dyadic local field that is a finite (ramified or unramified) extension of the usual 2-adic number field $Q_{2}$ (including $Q_{2}$ ). We let $\mathcal{O}$ stand for the ring of integers in $F, \mathscr{U}$ for the group of units, $\mathscr{P}$ for the unique maximal ideal, $\pi$ for a prime element generating $\mathscr{P}$, ord for the ordinal function, and || for the normalized multiplicative valuation in prime spot $\mathscr{P}$. The residue 
class field is a finite field of characteristic 2 and is therefore perfect. This means, in particular, that every unit $\varepsilon \equiv \mu^{2} \bmod \mathscr{P}$ for some unit $\mu$. The quadratic defect $\mathscr{D}(\alpha)$ of a field element $\alpha$ is the ideal generated by the element $\beta$ where $\alpha-\beta$ is a square and $|\beta|$ is minimal. If $\varepsilon \in \mathfrak{U}$, then

(i) $\mathscr{D}(\varepsilon)$ is one of the ideals: $0,4 \mathscr{O}, 4 \mathscr{P}^{-1} \cdots, \mathscr{P}^{3}, \mathscr{P}$;

(ii) $\mathscr{D}(\varepsilon)=4 \mathscr{O}$ if and only if $F(\sqrt{\varepsilon}) / F$ is a quadratic unramified extension;

(iii) suppose $\varepsilon=\eta^{2}+\alpha$ with $|4|<|\alpha|<1$ and ord $\alpha$ is odd, we have $\mathscr{D}(\varepsilon)=\alpha \mathcal{O}$.

Hensel's Lemma will frequently be applied, and usually we refer to it as the Local Square Theorem which states: "For any integer $\alpha \in \mathcal{O}, 1+4 \pi \alpha$ is a square."

If $\alpha, \beta$ are nonzero field elements, then $\alpha \beta \sim 1$ (or $\alpha \sim \beta$ ) means ord $\alpha \equiv \operatorname{ord} \beta \bmod 2$; otherwise, $\alpha \beta \sim \pi$.

A quadratic space $V$ over a field $F$ is simply a finite dimensional vector space endowed with a symmetric bilinear form $B$ (and its associated quadratic form $Q$ ). A lattice $L$ on $V$ is a finitely generated $O$-module in $V$ such that the subspace $F L$ spanned by $L$ equals $V$. The coefficient ideal of a vector $x$ in $V$ with respect to $L$ is

$$
\mathfrak{U}_{x}^{L}=\{\alpha \in F \mid \alpha x \in L\} \text {. }
$$

Vector $x$ is called maximal (primitive) in $L$ if $\mathfrak{A}_{x}^{L}=0$. A sublattice $M$ of $L$ splits if $M$ is an orthogonal direct summand, i.e. $L=M \perp N$ for some $N$. The $\mathcal{O}$-modules generated by the sets $B(L, L)$ and $Q(L)$ in $F$ are called the scale $\mathscr{S} L$ and the norm ideal $\mathscr{N} L$ respectively. Let $\mathfrak{A}$ be a fractional ideal, lattice $L$ is said to be $\mathfrak{A}$-modular if and only if $B(x, L)=\mathfrak{A}$ for every primitive vector $x \in L$. The norm group $\mathscr{G} L$ of $L$ is the additive subgroup of $F$ generated by $Q(L)$. This object is usually much finer than the norm ideal and it was first introduced by O'Meara to characterize completely isometric modular lattices. Theorem (O'Meara): Two modular lattices on the same quadratic space are isometric if and only if their scales and norm groups are equal. Hence, in particular, they are isometric if and only if they represent the same numbers in $F$. We shall obtain a result very analogous to this. We note here that even if $L$ is modular $Q(L)$ needs not equal $\mathscr{G} L$. O'Meara has shown [6] that if $L$ is modular with $\operatorname{dim} L \geqq 5$, then $Q(L)=\mathscr{G} L$. This was improved by Riehm (see [7], Th. 7.4) to $\operatorname{dim} L \geqq 4$. Notice that if $F$ is unramified (over $Q_{2}$ ) then the concepts of norm groups and norm ideals coincide since the maximal ideal $\mathscr{C} L$ contained in $\mathscr{G} L$ has always the same order parity as $\mathscr{N} L$. This reveals an important point as to why the unramified theory is very much simpler because $\mathscr{N} L$ is a far easier 
creature to contend with than $\mathscr{G} L$. A norm generator of $L$ is an element $a \in \mathscr{G} L$ such that $a \mathscr{O}=\mathscr{N} L$. The object $\mathscr{P}(\mathscr{C l} L)+2 \mathscr{S} L$ is called the weight ideal $\mathscr{W} L$ of $L$, and a scalar $b$ is called a weight generator if and only if $b \mathscr{O}=\mathscr{W} L$. An element $b \in \mathscr{G} L$ such that $a b \sim \pi$ and $|b|$ is the largest in $\mathscr{C} L$ is called a base generator of $L$ (following Riehm). It is well-known that $\mathscr{G} L=a \mathscr{O}^{2}+b \bigcirc$ where $b$ is either a base or a weight generator and $a$ is a norm generator. A base generator is often also a weight generator (e.g. when $\mathscr{W} L \supset 2 \mathscr{S} L$ ) and we shall use this letter $b$ indiscriminately. $L$ is a depleted modular lattice if $\mathscr{W} L=2 \mathscr{S} \mathrm{L}$. It was precisely this restriction to the size of $\mathscr{W} L$ that enabled the norm ideal to play a more dominant role and thereby facilitating us in our earlier solution of the integral equivalence problem over such lattices.

The symbol $A(\alpha, \beta)$ denotes a two dimensional unimodular (scale $=\infty$ ) lattice having basis $\{x, y\}$ such that $Q(x)=\alpha, Q(y)=\beta, B(x, y)=1$. Similarly, the symbol $\langle\alpha\rangle$ stands for an one dimensional lattice with a basis vector $\{x\}$ whose length is $Q(x)=\alpha$.

The set of all isometries of $V$ leaving $L$ stable is a subgroup $O(L)$ of the orthogonal group $O(V)$. Vectors $u, v \in L$ are integrally equivalent (symbolically $u \sim v$ ) if there exists an isometry $\sigma \in O(L)$ such that $\sigma(u)=v$. Our task is to determine necessary and sufficient conditions for integral equivalence when lattice $L$ is modular. Since the coefficient ideals and the lengths of $u$ and $v$ must clearly be the same for necessity, we shall henceforth take these vectors as being primitive in $L$ with common length $\delta$. By scaling (see [6]), we may assume $L$ is unimodular. Furthermore, since the depleted case has been settled we may assume, whenever necessary, that $\mathscr{\mathscr { V }} L \supset 2 \bigcirc$ which implies, in particular, ord $(\mathscr{N} L)+$ ord $(\mathscr{W} L)$ is odd. Also, if $\operatorname{dim} L \geqq 3$, then $L$ represents every weight (base) generator.

Finally, we associate to every maximal vector $x \in L$ its characteristic set

$$
\mathfrak{M}_{x}=\{z \in L \mid B(x, z)=1\} \text {. }
$$

The numbers represented by this set will be an important invariant needed to classify integrally equivalent vectors.

\section{Binary case.}

Definition. Let $L$ be binary unimodular and $u, v \in L$. We say $u, v$ are of the same parity if and only if for all pairs $(\bar{u}, \bar{v})$ of vectors in $L$ such that $\bar{u} \in \mathfrak{M}_{u}, \bar{v} \in \mathfrak{M}_{v}$ we have

$$
Q(\bar{u}) \equiv Q(\bar{v}) \bmod \omega \mathcal{O}
$$

where $\omega=\max \{2, \delta\}$. (Of course, maximum is taken in the sense of 
their valuations.)

We have proved in [2] the following result.

THEOREM 2.1. Let $L$ be any binary unimodular lattice. Then, $u \sim v$ if and only if they are of the same parity.

Proposition 2.2. Suppose $L$ is binary unimodular with $\mathscr{W} L \supset 2 \bigcirc$, then $u \sim v$ always.

Proof. By ([6], 93:10) we have $L \cong A(a, b)$ where $a$ and $b$ are norm and weight generator respectively. Hence, $O(L)=O(F L)$ by ([7], Lemma 3.5). Now $u \sim v$ by Witt's Theorem.

\section{Classification of vectors.}

Definitions. A maximal (primitive) vector $x$ in $L$ is $\mathscr{N}$-regular (resp. $\mathscr{G}$-regular) if and only if $\mathscr{N}\left(\langle x\rangle^{\perp}\right)=\mathscr{N} L\left(\operatorname{resp} . \mathscr{G}\left(\langle x\rangle^{\perp}\right)=\mathscr{G} L\right)$, where

$$
\langle x\rangle^{\perp}=\{z \in L \mid B(x, z)=0\} .
$$

Otherwise, $x$ is $\mathscr{N}$-irregular (resp. G-irregular).

Again, putting $\omega=\max \{2, \delta\}$, we call $u$ a vector of Type I if $\mathscr{N}\left(\langle u\rangle^{\perp}\right) \subseteq \omega O$; otherwise, $u$ is of Type II.

REMaRK. If $F$ is unramified, then the concepts of $\mathscr{N}$-regularity and $\mathscr{G}$-regularity coincide.

Definition. Suppose $L$ is unimodular with $\operatorname{dim} L=2 n, n \geqq 1$. Then, there exists a splitting

$$
L=L_{1} \perp \cdots \perp L_{n}
$$

where $L_{i} \cong A\left(a_{i}, \gamma_{i}\right)$ with $a_{i} \mathscr{O}=\mathscr{N} L, \gamma_{i} \in \mathscr{W} L_{i} \cong \mathscr{W} L$ for $i=$ $1, \cdots, n$. Such a splitting is called a quasi-canonical splitting ${ }^{1}$.

It is quite clear that if $Q(u)=\delta \notin \mathscr{U}$ and $\operatorname{dim} L$ is odd, then $u$ is $\mathscr{N}$-regular always. Also, $u$ is $\mathscr{N}$-regular whenever $\delta$ is a norm generator and $\operatorname{dim} L$ is even. The $\mathscr{N}$-irregular vectors are characterized as follows: (i) Assume $\operatorname{dim} L \geqq 3$. If $u$ is $\mathscr{N}$-irregular, then for every $\bar{u} \in \mathfrak{M}_{u}, Q(\bar{u})$ is a norm generator. The converse is true provided $L$ is not totally improper (i.e. $\mathscr{N} L \neq 2 \mathscr{S} L=20$ ). (ii) Let $\operatorname{dim} L=2 n, n \geqq 1$. For every quasi-canonical splitting

$$
L={ }_{i=1}^{n} L_{i}, L_{i} \cong A\left(a_{i}, \gamma_{i}\right)=\mathcal{O} x_{i}+\mathcal{O} y_{i}, 1 \leqq i \leqq n,
$$

1 The existence is seen by applying ([6], 93:12 and 93:18) and O'Meara's op-transformations (see [5]). 
INTEGRAL EQUIVALENCE OF VECTORS OVER LOCAL MODULAR LATTICES 531

we put

$$
u=\sum\left(\alpha_{i} x_{i}+\beta_{i} y_{i}\right), \alpha_{i}, \beta_{i} \in \mathcal{O} .
$$

Then, $u$ is $\mathscr{N}$-irregular implies all the $\beta_{i}$ 's are unitary. Again, the converse holds for $L$ not totally improper.

Condition (D). (Assume $\operatorname{dim} L \geqq 3$, and $\mathscr{\mathscr { V }} L \supset 20$ ). An element $\alpha$ in $\mathscr{G} L$ is said to satisfy condition (D) provided the quadratic defect satisfies the inequality

$$
\mathscr{D}(\alpha \alpha) \subset \mathscr{N} L \mathscr{W} L
$$

for every norm generator $a \in \mathscr{G} L$.

Lemma 3.1. Suppose $\delta \in \mathscr{G} L$ and $\delta \mathscr{O} \neq \mathscr{N} L$. Then, if there exists one norm generator $a^{\prime}$ such that $\mathscr{D}\left(\delta a^{\prime}\right) \subset \mathscr{N} L \mathscr{W} L$, we have $\delta$ satisfying condition (D).

Proof. Write $\mathscr{G} L=a^{\prime} \mathcal{O}^{2}+b \mathcal{O}$ for the given $a^{\prime}$ and an arbitrary

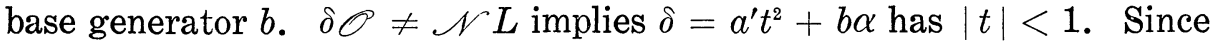
$a^{\prime} b \sim \pi$ we see $|\alpha|<1$ by the assumption that $\mathscr{D}\left(\delta a^{\prime}\right) \subset \mathscr{N} L \mathscr{W} L$. The rest is computational.

Proposition 3.2. Let $u$ be an $\mathscr{N}$-regular vector with length $\delta$ satisfying condition (D). Then $u$ is also $\mathscr{G}$-regular if and only if there exists a vector $\bar{u} \in \mathfrak{M}_{u}$ such that $Q(\bar{u}) \in \mathscr{P} \mathscr{W} L$.

Proof. Since $\delta$ satisfies condition (D), we have the implicit assumptions of $\operatorname{dim} L \geqq 3$ and $\mathscr{\mathscr { V }} L \supset 20$. Therefore, $\delta$ is not a norm generator since otherwise $\delta+b$ is a norm generator also (here $b$ is any base generator) and

$$
\mathscr{D}(\delta(\delta+b))=\delta b \mathcal{O}=\mathscr{N} L \mathscr{W} L,
$$

implying that $\delta$ does not satisfy condition (D). Putting $L=K \perp M$ where

$$
K=\mathcal{O} u+\mathcal{O} \bar{u} \cong A(\delta, Q(\bar{u})) \text { with } Q(\bar{u}) \in \mathscr{P} \mathscr{W} L,
$$

it is quite clear that $\mathscr{N} M=\mathscr{N} L$. Write

$$
\mathscr{G} M=a_{m} \mathscr{O}^{2}+b_{m} \mathscr{O} \text { and } \mathscr{G} K=a_{k} \mathscr{O}^{2}+b_{k} \mathscr{O} \text {. }
$$

But,

$$
\mathscr{W} L=\sum_{\gamma} a_{m}^{-1} \mathscr{D}\left(a_{m} \gamma\right)
$$

where $\gamma$ runs through the set $\left\{b_{m}, a_{k}, b_{k}\right\}$ (see page 31, [7]). Now, 
$\mathscr{D}\left(a_{m} a_{k}\right) \subset \mathscr{N} L \mathscr{W} L$ since $\delta$ satisfies condition $(\mathrm{D})$ and $Q(\bar{u}) \in \mathscr{P} \mathscr{\mathscr { V }} L$. If $|\delta| \geqq|\mathscr{W} L|$, then

$$
b_{k} \mathscr{O}=\delta^{-1} \mathscr{D}(\delta Q(\bar{u}))+2 \mathscr{O} \subset \mathscr{W} L .
$$

On the other hand, $\delta \in \mathscr{P} \mathscr{W} L$ implies $b_{k} \mathscr{O} \subset \mathscr{W} L$ since $L$ is not depleted by hypothesis. Thus, $\mathscr{W} L=\mathscr{W} M$ proving $u$ is G-regular.

Conversely, assume $\mathscr{G}\left(\langle u\rangle^{\perp}=\mathscr{G} L\right.$. Suppose the contrary is true, i.e. every $u^{\prime} \in \mathfrak{M}_{u}$ is such that $Q\left(u^{\prime}\right) \notin \mathscr{P} \mathscr{W} L$. Putting $L=$ $\left(\mathcal{O} u+\mathcal{O} u^{\prime}\right) \perp T$, we see that $\mathscr{N} T=\mathscr{N} L$ since $u$ is $\mathscr{N}$-regular with $\delta$ not being a norm generator. We also have,

$$
T=\left\{\begin{array}{l}
\langle a\rangle \perp \cdots=\varnothing x \perp \cdots \\
A(a, \cdots) \perp \cdots=(x \odot+\cdots) \perp \cdots
\end{array}\right.
$$

Suppose $Q\left(u^{\prime}\right)$ is already a norm generator, we apply $o p\left(u^{\prime}\right)=u^{\prime} \perp \varepsilon x$ where $\varepsilon$ is an unit such that

$$
Q(\varepsilon x) \equiv Q\left(u^{\prime}\right) \bmod \mathscr{W} L .
$$

Now, $Q\left(o p\left(u^{\prime}\right)\right)$ lies in $\mathscr{W} L$. On the other hand, if $\left|Q\left(u^{\prime}\right)\right|<|\mathscr{N} L|$, by applying $o p\left(u^{\prime}\right)=u^{\prime} \perp x$ we have made $o p\left(u^{\prime}\right)$ a norm generator and furthermore,

$$
L=\left(\mathscr{O} u+\mathscr{O}\left(o p\left(u^{\prime}\right)\right)\right) \perp T^{\prime}, \mathscr{N} T^{\prime}=\mathscr{N} L .
$$

Therefore, in either case we know that by applying op-transformations, at most twice if necessary, there exists a vector $\bar{u} \in \mathscr{Y}_{u}$ with $\operatorname{ord} Q(\bar{u})=\operatorname{ord}(\mathscr{W} L)$. Let $Q(\bar{u})=b$ and write

$$
L=K \perp M
$$

again as above. Then, $\mathscr{N} M=\mathscr{N}^{*} L$ and

$$
\langle u\rangle^{\perp} \cong\langle d K \delta\rangle \perp M .
$$

where $d K$ is the discriminant of $K$. Now, writing $a_{m}=a$, it is easy to see that $\mathscr{D}(a \delta) \subset a b \mathcal{O}$ implies also that $\mathscr{D}(a \delta d K) \subset a b \mathcal{O}$. Hence, $\mathscr{G} M=\mathscr{G} L$. This means, in particular, that $M$ represents every weight (base) generator of $G L$ whenever $\operatorname{dim} M \geqq 3$. By applying: $o p$-transformations, if necessary, and by the perfectness of the residue class field, we can find an $\bar{u} \in \mathfrak{M}_{u}$ with $Q(\bar{u}) \in \mathscr{P} \mathscr{W} L$ and so we are done except for $\operatorname{dim} M=1,2$. But $\operatorname{dim} M=1$ is not possible since $L$ is not depleted. (Referring to $(*)$ above, one sees immediately that since $\delta$ does not satisfy condition (D) -nor does $\delta d K-\operatorname{dim} M=1$ would imply $u$ is $\mathscr{G}$-irregular contradicting hypothesis.) Finally, suppose $\operatorname{dim} M=2$. We express

$$
M \cong A\left(a,-\alpha a^{-1}\right)
$$


where $\left|-\alpha a^{-1}\right|=|\mathscr{W} L|$, (see [6], 93:10\& 93:17). Again, it is not difficult to see that there is a suitable op-transformation such that $o p(\bar{u}) \in \mathfrak{M}_{u}$ with length contained in $\mathscr{P} \mathscr{V} L$, contradicting the initial assumption.

CoROLlaRY 3.3. If $u$ is a G-regular vector with length $Q(u)=\delta$ satisfying condition (D), then (i) $\operatorname{dim} L \geqq 4$, and (ii) for every $\bar{u} \in \mathfrak{M}_{u}$, $\mathscr{G}(\mathcal{O} u+\mathscr{O} \bar{u})^{\perp}=\mathscr{G} L$.

Proposition 3.4. Let $\operatorname{dim} L \geqq 3$ and $\delta$ not satisfying condition (D). Then, $u \sim v$ if and only if $\langle u\rangle^{\perp} \cong\langle v\rangle^{\perp}$.

Proof. We may assume $\delta$ is not a norm generator by Proposition 2 in [2]. Therefore, $\mathscr{D}\left(\delta a^{\prime}\right)=\mathscr{N} L \mathscr{W} L$ for each norm generator $a^{\prime} \in \mathscr{G} L$. Putting $\mathscr{G} L=a^{\prime} \mathscr{O}^{2}+b \mathscr{O}$ for some base generator $b$, we have

$$
\delta=a^{\prime} t^{2}+b \varepsilon,|\varepsilon|=1,|t|<1 .
$$

Case I. Suppose both $u$ and $v$ are $\mathscr{N}$-regular, then there is $\bar{u} \in \mathfrak{M}_{u}$ with $Q(\bar{u}) \in b \mathcal{O}$. Let $K_{u}=\mathscr{O} u+\mathscr{O} \bar{u}, M_{u}=K_{u}^{\perp}$. Then, $\mathscr{N} M_{u}=\mathscr{N} L$. Since $a^{\prime} b \sim \pi$, ord $\delta \leqq \operatorname{ord} b$. Let $\sigma:\langle u\rangle^{\perp} \rightarrow\langle v\rangle^{\perp}$ be the given isometry and $\sigma\left(M_{u}\right)=M_{v}$. Then $M_{v}$ splits.

$$
L=K_{v} \perp M_{v} \text { with } K_{v}=\varnothing v+\wp \bar{v} \cong A(\delta, Q(\bar{v}))
$$

for some $\bar{v} \in M_{v}$. We claim

$$
Q(\bar{v}) \in \delta \mathscr{O}
$$

Suppose not. Put

$$
Q(\bar{v})=a^{\prime} s^{2}+b r \text { with }\left|a^{\prime} s^{2}\right|>|b| .
$$

Then,

$$
\delta Q(\bar{v})=\left(a^{\prime} t s\right)^{2}+a^{\prime} s^{2} b \varepsilon+b^{2} \varepsilon r+a t^{2} b r .
$$

Clearly, we may assume that ord $s<$ ord $t$. But,

$$
d K_{u} \cong d K_{v} \Longrightarrow \mathscr{D}\left(-d K_{u}\right)=\mathscr{D}\left(-d K_{v}\right) .
$$

Now, $-d K_{u}$ has quadratic defect contained in $\delta b \odot$. On the other hand, by direct computations, we see

$$
\mathscr{D}\left(-d K_{v}\right)=Q(\bar{v}) b \mathscr{O} \supset \mathscr{D}\left(-d K_{u}\right) .
$$

This is a contradiction so the claim is true. Hence, $\mathscr{N} K_{u}=\mathscr{N} K_{v}=$ $\delta \mathcal{O}$ and we see readily that $\mathscr{G} K_{u}=\mathscr{G} K_{v}$. By Witt's Theorem and 
O'Meara's Theorem on isometry of modular lattices, $K_{u} \cong K_{v}$. Finally, $u \sim v$ follows from another application of Proposition 2, [2].

Case II. Suppose both are $\mathscr{N}$-irregular. Let $\bar{u} \in \mathfrak{M l}_{u}$ be arbitrary and $K_{u}, M_{u}, M_{v}, K_{v}=\mathcal{O} v+\mathcal{O} \bar{v}$ for some $\bar{v} \in \mathfrak{M}_{v}$ as before. But now, $Q(\bar{u})$ and $Q(\bar{v})$ are norm generators for $\mathscr{G} L . F K_{u}$ is isometric to $F K_{v}$ by Witt. It is an easy computation to check that the sublattices $K_{u}$ and $K_{v}$ are not depleted; indeed, $\mathscr{G} K_{u}=\mathscr{G} K_{v}=\mathscr{G} L$. Hence, $u \sim v$ by Proposition 2.2.

REMARK. It can be shown that when $\operatorname{dim} L=3$ and if $\delta$ does not satisfy condition (D), then $u \sim v$ always provided $\delta \notin \mathscr{C}$. In proving this fact, we show that $\langle u\rangle^{\llcorner} \cong\langle v\rangle^{\perp}$ by using O'Meara's Theorem $93: 28,[6]$.

Proposition 3.5. Suppose $\operatorname{dim} L \geqq 3$ and both $u$ and $v$ are Type I vectors. Then, $u \sim v$ if and only if (i) $u, v$ are of the same parity, and (ii) $\langle u\rangle^{\perp} \cong\langle v\rangle^{\perp}$.

Proof. The case of $\delta$ being an unit is obvious. So, let $\delta \notin \mathscr{U}$. Hence, choose any $\bar{u} \in \mathfrak{M}_{u}$ and put

$$
K_{u}=\mathcal{O} u+\mathcal{O} \bar{u}, T_{u}=K_{u}^{\perp} .
$$

Suppose $\sigma:\langle u\rangle^{\perp} \rightarrow\langle v\rangle^{\perp}$ is the given isometry. Then, $\sigma\left(T_{u}\right)=T_{v}$ splits and we have

$$
L=K_{v} \perp T_{v}
$$

where $K_{v}=\mathcal{O} v+\mathcal{O} \bar{v}$ for some $\bar{v} \in \mathfrak{M}_{v}$. If $|\delta| \leqq|2|$, then (i) implies $\mathscr{G} K_{v}=\mathscr{G} K_{u}$ so that $K_{u} \cong K_{v}$ by Witt and O'Meara and therefore $u \sim v$ follows from Theorem 2.1. Otherwise, define the mapping $\phi: F K_{u} \rightarrow F K_{v}$ by: $\phi(u)=v, \phi(u-\delta \bar{u})=\mu(v-\delta \bar{v})$ where

$$
\mu^{2}=\frac{1-\delta Q(\bar{u})}{1-\delta Q(\bar{v})}
$$

Now, again condition (i) implies that

$$
\mu^{2} \equiv 1 \bmod \delta^{2} \mathcal{O} \text {. }
$$

It is easily checked that $\phi$, in fact, maps $K_{u}$ onto $K_{v}$ and we are done.

4. Main results. We recall that to every maximal vector $x$ in $L$, there is associated with it a characteristic subset $\mathfrak{M}_{x}$ of the lattice

$$
\mathfrak{M}_{x}=\{z \in L \mid B(x, z)=1\} .
$$


A central result given below states that $u$ is integrally equivalent to $v$ if and only if $\mathfrak{M}_{u}$ and $\mathfrak{M}_{v}$ represent the same field elements when the dimension of the given lattice is sufficiently large. This theorem may be viewed (for $\operatorname{dim} L$ large enough) as an analogue to the wellknown theorem on the integral classification of modular quadratic forms over local fields.

THEOREM 4.1. Let $L$ be an unimodular lattice over a dyadic local field of characteristic zero, and that $\operatorname{dim} L \neq 4,5,6$. Then, two maximal vectors $u$ and $v$ in $L$ are integrally equivalent if and only if $Q(u)=\delta=Q(v)$ and $Q\left(\mathfrak{M R}_{u}\right)=Q\left(\mathfrak{M}_{v}\right)$.

Proof. Necessity is obvious. As for sufficiency we proceed in several steps.

1. Pick any $\bar{u} \in \mathfrak{M}_{u}, \bar{v} \in \mathfrak{M}_{v}$ such that $Q(\bar{u})=Q(\bar{v})$. Then,

$$
\begin{aligned}
& \mathfrak{M}_{u}=\bar{u}+\mathcal{O}(u-\delta \bar{u}) \perp T_{u}=\bar{u}+\langle u\rangle^{\perp} \\
& \mathfrak{M}_{v}=\bar{v}+\mathcal{O}(v-\delta \bar{v}) \perp T_{v}=\bar{v}+\langle v\rangle^{\perp} .
\end{aligned}
$$

Hence, $Q\left(\langle u\rangle^{\perp}\right)=Q\left(\langle v\rangle^{\perp}\right) \bmod 2 \mathcal{O}^{\circ} \quad$ (i.e. for every $z \in\langle u\rangle^{\perp}, \exists w \in\langle v\rangle^{\perp}$ such that $Q(z) \equiv Q(w) \bmod 20)$ Therefore, the norm groups are equal

$$
\mathscr{G}=\mathscr{G}\left(\langle u\rangle^{\perp}\right)=\mathscr{G}\left(\langle v\rangle^{\perp}\right) .
$$

It is also clear that $F\langle u\rangle^{\perp} \cong F\langle v\rangle^{\perp}$ and $F T_{u} \cong F T_{v}$.

2. Suppose $\operatorname{dim} L \geqq 9$ so that $\operatorname{dim} T_{u}=\operatorname{dim} T_{v} \geqq 7$. Then, it is well-known (see [6], 93:18) that

$$
T_{u} \cong A(0,0) \perp A(0,0) \perp \cdots .
$$

Take a norm generator $a^{\prime}$ and $a$ base (weight) generator $b$ for $\mathscr{G}\left(\langle u\rangle^{\perp}\right)$. So, $\mathscr{G}\left(\langle u\rangle^{\perp}\right)=a^{\prime} \mathscr{O}^{2}+b \mathcal{O}$. Therefore,

$$
\langle u\rangle^{\perp} \cong A(0,0) \perp A(0,0) \perp K_{u}
$$

for some $K_{u}$. But now,

$$
\mathscr{G}\left(K_{u}^{\circ}\right)=2 \mathscr{O}+\mathscr{G}\left(K_{u}^{\circ}\right)=\mathscr{G}\left(\langle u\rangle^{\perp}\right)
$$

so that $a^{\prime}, b$ lie in $\mathscr{G}\left(K_{u}^{\circ}\right)$. (Here $K_{u}^{\circ}=\left\{x \in K_{u} \mid B\left(x, K_{u}\right) \subseteq \mathcal{O}\right\}$.) Hence, by ([6], 93:13), we have

$$
\langle u\rangle^{\perp} \cong A\left(a^{\prime}, 0\right) \perp A(b, 0) \perp K_{u} .
$$

This means there exists a Jordan decomposition

$$
\langle u\rangle^{\perp}=W_{1} \perp W_{2}
$$

where $\mathscr{G} W_{1}=\mathscr{G}\left(\langle u\rangle^{\perp}\right)$ and $W_{2} \cong \mathscr{O}(u-\delta \bar{u})$. 
3. Let $\operatorname{dim} L \geqq 7$. Just adjoin an hyperbolic plane $H$ to $L, H \cong$ $A(0,0)$. Now, apply step (2) and we have

$$
\langle u\rangle^{\perp} \perp H=W_{1}^{*} \perp W_{2}^{*}
$$

with $\mathscr{G} W_{1}^{*}=\mathscr{G}\left(\langle u)^{\perp}\right)$ and $W_{2}^{*} \cong \mathscr{Q}(u-\delta \bar{u})$. But, $\operatorname{dim} W_{1}^{*} \geqq 7$ here so that $W_{1}^{*}$ admits a splitting

$$
W_{1}^{*} \cong A(0,0) \perp A(0,0) \perp W_{1}^{\prime} .
$$

Clearly, $\mathscr{G} W_{1}^{\prime}=\mathscr{G} W_{1}^{*}=\mathscr{G}\left(\langle u\rangle^{\perp}\right)$. Upon cancelling the hyperbolic plane, we obtain

$$
\langle u\rangle^{\perp} \cong W_{1}^{\prime} \perp W_{2}^{*} \text {. }
$$

Similarly for $\langle v\rangle^{\perp}$. Hence, $\langle u\rangle^{\perp} \cong\langle v\rangle^{\perp}$.

4. By (2) and (3), we put $L_{u} \perp P_{u}=L=L_{v} \perp P_{v}$, where $P_{u} \cong P_{v}$ have norm groups equal to $\mathscr{G}$, and $L_{u}=\mathscr{O} u+\mathcal{O} u^{\prime}, L_{v}=\mathscr{O} v+\mathcal{O} v^{\prime}$. Let $v^{*} \in \mathfrak{M}_{v}$ such that $Q\left(v^{*}\right)=Q\left(u^{\prime}\right)$. Hence, we have

$$
v^{\prime}=v^{*}+w, w \in\langle v\rangle^{\llcorner} .
$$

Therefore,

$$
Q\left(u^{\prime}\right)=Q\left(v^{\prime}\right)+\alpha, \quad \text { for some } \alpha \text { in } \mathscr{G} \text {. }
$$

On the other hand, we have

$$
P_{v} \cong A(0,0) \perp R_{v} \quad \text { where } \mathscr{G} R_{v}=\mathscr{G} \text { still! }
$$

By ([6], $93: 13)$,

$$
P_{v} \cong A(\alpha, 0) \perp R_{v}=(\mathscr{O} y+\cdots) \perp R_{v} .
$$

Applying the op-transformation: $v^{\prime} \rightarrow o p\left(v^{\prime}\right)=v^{\prime} \perp y$, we see that

$$
L_{v}^{\prime}=\mathscr{O} v+\mathscr{O}\left(o p\left(v^{\prime}\right)\right) \text { splits } L
$$

with $P_{v}^{\prime}$ as its orthogonal complement and furthermore, $\mathscr{G} P_{v}^{\prime}$ still equals $\mathscr{G}$. Now, $u \sim v$ is clear.

5. When $\operatorname{dim} L$ is less than 4, the proof of the theorem is entirely trivial.

COROLLARY 4.2. Let $L$ be an unimodular lattice with arbitrary dimension, $u$ and $v$ be to two maximal vectors in $L$ having the same length. If $Q\left(\mathfrak{M}_{u}\right)=Q\left(\mathfrak{M}_{v}\right)$, then, $\langle u\rangle^{\perp} \cong\langle v\rangle^{\perp}$.

Proof. By adjoining a suitable number of $A(0,0)$ 's and calling the enlarged lattice $L^{\prime}$, we have $u$ integrally equivalent to $v$ over $L^{\prime}$. Hence, 


$$
\langle u\rangle^{\perp}\left(\text { in } L^{\prime}\right) \cong\langle v\rangle^{\perp}\left(\text { in } L^{\prime}\right)
$$

But,

$$
\langle u\rangle^{\perp}\left(\text { in } L^{\prime}\right)=\langle u\rangle^{\perp}(\text { in } L) \perp A(0,0) \perp \cdots \perp A(0,0)
$$

and similarly for $\langle v\rangle^{\perp}$. Now, cancel out the $A(0,0)$ 's .

COROLlaRY 4.3. Let $L$ be unimodular having arbitrary dimension, $\delta \in 2 \mathcal{O}$, and $Q\left(\mathfrak{M}_{u}\right)=Q\left(\mathfrak{M}_{v}\right)$. Then, $u \sim v$ always.

Proof. Choose any $\bar{u} \in \mathfrak{M}_{u}$ and $\bar{v} \in \mathfrak{M}_{v}$ such that $Q(\bar{u})=Q(\bar{v})$, and put

$$
L_{u}=\varnothing u+\varnothing \bar{u}, P_{u}=L_{u}^{\perp}, L_{v}=\varnothing v+\varnothing \bar{v}, P_{v}=L_{v}^{\perp} .
$$

Then, $F P_{u} \cong F P_{v}$. Since $\delta$ lies in 20 , it is clear that $\mathscr{G} P_{u}=\mathscr{C} P_{v}$. The rest is obvious.

THeOREM 4.4. Let $\operatorname{dim} L=4,5$ and $Q\left(\mathfrak{M}_{u}\right)=Q\left(\mathfrak{M}_{v}\right)$. If there exists a vector $\bar{u} \in \mathfrak{M}_{u}$ such that $(\mathcal{O} u+\mathscr{O} \bar{u})^{\perp}$ is isotropic, then $u \sim v$.

We shall first prove a lemma.

LEMMA 4.5. Under the same hypothesis as in the theorem except $\operatorname{dim} L$ may be 6 , there exist then vectors $x_{u} \in \mathfrak{M}_{u}, x_{v} \in \mathfrak{M}_{v}$ such that $Q\left(x_{u}\right)=Q\left(x_{v}\right)$ and moreover, by denoting $L_{u}=\mathcal{O} u+\mathcal{O} x_{u}$ and $L_{v}=$ $\mathcal{O} v+\mathcal{O} x_{v}$, we will have $F L_{u}^{\frac{1}{u}} \cong F L_{v}^{\lrcorner}$are isotropic spaces, and $\mathscr{N}\left(L_{u}^{\perp}\right)=\mathscr{N}\left(\langle u\rangle^{\perp}\right)=\mathscr{N}\left(L_{v}^{\perp}\right)$.

Proof. The case of $\delta \in \mathscr{U}$ is quite obvious. Let $\bar{u}$ be the given vector, we put $K_{u}=\mathscr{O} u+\mathscr{O} \bar{u}$. If $u$ is a Type II vector (hence so is $v$ ), then $\mathscr{N}\left(K_{u}^{\perp}\right)$ already equals $\mathscr{N}\left(\langle u\rangle^{\perp}\right)$ and everything is clear. So let both be Type I vectors. By a suitable op-transformation, we may assume $\mathscr{N}\left(K_{u}^{\perp}\right)=\mathscr{N}\left(\langle u\rangle^{\perp}\right)$. Pick $\bar{v}$ from $\mathfrak{M}_{v}$ with $Q(\bar{v})=Q(\bar{u})$, and denote $K_{v}=\mathcal{O} v+\mathcal{O} \bar{v}$. By Witt's Theorem, $F K_{v}^{\perp}$ is isotropic. Hence, $K_{v}^{\perp}$ has a splitting of the form

$$
K_{v}^{\perp}=A(s, 0) \perp \cdots=(\mathscr{O} x+\mathscr{O} z) \perp \cdots .
$$

We know that

$$
\langle v\rangle^{\perp}=K_{v}^{\perp} \perp \mathcal{O}(v-\delta \bar{v}) .
$$

Apply $o p(x)=x \perp(v-\delta \bar{v})$, then $K_{v}^{\perp}$ becomes $(\mathscr{O}(o p(x))+\mathscr{O} z) \perp \cdots$ and call this $T_{v}^{\perp}$ where $T_{v}=\mathscr{O} v+\mathcal{O} w$ for some $w \in \mathfrak{M}_{v}$. In fact,

$$
w=\bar{v}+\alpha(v-\delta \bar{v})+\beta x+\gamma z, \alpha, \beta, \gamma \in \mathcal{O} .
$$


Using the fact that $w$ is orthogonal to both $z$ and $o p(x)$, one deduces $\beta=0$ and $\gamma=(1-\alpha \delta) D$, where $D$ is the discriminant of $K_{v}$. Consequently,

$$
Q(w)=Q(\bar{v})+\alpha^{2} \delta D-2 \alpha D .
$$

Here, $\alpha$ can be quite arbitrary so that by choosing order of $\alpha$ to be sufficiently large, we see that $T_{v} \cong K_{v}$. But now, $T_{v}^{\perp}$ is easily seen to have its norm ideal equals to $\mathscr{N}\left(\langle v\rangle^{\perp}\right)=\mathscr{N}\left(\langle u\rangle^{\perp}\right)$, because if $|\delta|\left\langle\left|\mathscr{N}\left(\langle v\rangle^{\perp}\right)\right|\right.$ then $K_{v}^{\perp}$ already has norm equal to $\mathscr{N}\left(\langle v\rangle^{\perp}\right)$. The existence of such vectors $x_{u}$ and $x_{v}$ is now clear.

Proof of the theorem. Let $L_{u}=\mathscr{O} u+\mathscr{O} x_{u}$ and $L_{v}=\mathscr{O} v+\mathcal{O} x_{v}$ enjoy the properties as stated in the lemma. We put $D=d L_{u}=d L_{v}$. Suppose, for the moment, that $L$ is quarternary. Then, we write

$$
L_{u}^{\perp}=A\left(a_{u}, 0\right), \quad L_{v}^{\perp}=\mathscr{O} x+\mathscr{O} z=A\left(a_{v}, 0\right)
$$

where $a_{u}$ and $a_{v}$ are norm generators for $\mathscr{G}\left(\langle u\rangle^{\perp}\right)$. If $\mathscr{W}\left(\langle u\rangle^{\perp}\right)=$ 20 , then it is easy to see that $L_{u}^{\perp} \cong L_{v}^{\perp}$ and so $u \sim v$. Therefore, $\mathscr{D}\left(a_{u} \delta D\right)$ must equal $a_{u} \mathcal{O} \mathscr{W}\left(\langle u\rangle^{\perp}\right) ! \quad$ As in the lemma, since $a_{u} \in Q\left(\langle v\rangle^{\perp}\right)$ we have

$$
a_{u}=A^{2} a_{v}+B^{2} \delta D+2 A^{\prime}, \quad|A|=1, B, A^{\prime} \in \mathcal{O} .
$$

Applying the $o p$-transform, $o p(x)=x \perp B A^{-1}\left(v-\delta x_{v}\right)$ one sees by direct computations that

$$
L=\left(\mathscr{O} v+\mathscr{O}\left(x_{v} \perp D B A^{-1} z\right)\right) \perp(\mathscr{O}(o p(x))+\mathscr{O} z) .
$$

(The choice of $x_{v} \perp D B A^{-1} z$ corresponds to the choice of $\alpha$ equals zero in the proof of the lemma.) Now, observe that the first term on the right-hand-side is isometric to $L_{u}$ and the second term is isometric to $L_{u}^{\perp}$ because

$$
Q(o p(x)) \equiv a_{u} A^{2} \bmod 2 \mathcal{O}, \text { with } A \in \mathscr{U} .
$$

Hence, $u \sim v$.

Now, let $\operatorname{dim} L=5$. By proving a result similar to Lemma 4.5, we may assume $\left(\mathscr{O} u+\mathscr{O} x_{u}\right)^{\perp}$ has norm group equal to $\mathscr{G}\left(\langle u\rangle^{\perp}\right)$. But, $\left(\mathscr{O} v+\mathcal{O} x_{v}\right)^{\perp}$ will not, in general, simultaneously enjoy this same property.

Let us call $P_{u}=L_{u}^{\perp}, P_{v}=L_{v}^{\perp}, \mathscr{G}\left(\langle u\rangle^{\perp}\right)=\mathscr{G}=\bar{a} \mathscr{O}^{2}+\bar{b} \mathscr{O}$; the discriminant of $P_{u}$ we denote by $d$ and therefore by ([6], 93:18)

$$
\begin{aligned}
& P_{u} \cong\langle-d\rangle \perp A(\bar{b}, 0) \\
& P_{v} \cong\langle-d\rangle \perp A\left(b^{\prime}, 0\right),
\end{aligned}
$$


where $b^{\prime}$ is a weight generator for $\mathscr{G} P_{v}$, which we may assume to be of having larger order than that of $\bar{b}$ because otherwise, $P_{u} \cong P_{v}$ already.

Suppose the component $A\left(b^{\prime}, 0\right)$ is adapted to a basis $\mathscr{O} x+\mathscr{O} z$ again. Take $o p(x)=x \perp\left(v-\delta x_{v}\right)$. If $D$ still denotes the discriminant of $L_{v}=\mathscr{O} v+\mathscr{O} x_{v}$, it is readily seen that

$$
L=\left(\mathscr{O} v+\mathscr{O}\left(x_{v} \perp D z\right)\right) \perp\langle-d\rangle \perp(\mathscr{O}(o p(x))+\mathscr{O} z),
$$

where the first term on the right side again is isometric to $L_{u}$. Because of the assumption that $\left|b^{\prime}\right|<|\bar{b}|$, we must have $\mathscr{D}\left(-d\left(b^{\prime}+\delta D\right)\right)$ equal to $\mathscr{W}\left(\langle u\rangle^{\perp}\right)$. Hence,

$$
P_{u} \cong\langle-d\rangle \perp(\mathcal{O}(o p(x))+\mathcal{O} z) .
$$

The rest is obvious.

REMARKs. (i) It follows from the proofs of the theorem and the lemma that if either $\delta \in \mathscr{W}\left(\langle u\rangle^{\perp}\right) \mathscr{P}$ or $\mathscr{W}\left(\langle u\rangle^{\perp}\right) \neq 20$, then $u \sim v$ regardless of the dimension and the existence of the vector $\bar{u}$ with the stated property.

(ii) A 4-dimensional unimodular lattice with given discriminant assumes two possible forms (either $J$ or $K$ in $93: 18$ of [6]). By a result of Riehm (Theorem 7.4, [7]) it is known that such a lattice represents every element of its norm group. Now, employing the same notations as in the proof of the last theorem, it is readily seen that we may assume when $\operatorname{dim} L=6$ (as in the $\operatorname{dim} L=5$ case) that $\left(\mathcal{O} u+\mathscr{O} x_{u}\right)^{\perp}$ has norm group equal to $\mathscr{G}$ already. Hence, the proof of Theorem 4.4 still goes through if $P_{u}$ takes the " $J$-form". (It is easily seen that $P_{u}$ takes the " $J$-form" if and only if $P_{v}$ does so since the spaces on which they sit are isometric.)

(iii) Theorem 4.4 also goes through when $\operatorname{dim} L=6$ and when both $u$ and $v$ are $\mathscr{N}$-irregular vectors.

(iv) Finally, we remark that Theorem 4.1 remains valid if and only if the characteristic sets satisfy the somewhat weaker property: For each $\bar{u} \in \mathfrak{M}_{u}$, there is a vector $\bar{v} \in \mathscr{C l}_{v}$ with

$$
Q(\bar{u}) \equiv Q(\bar{v}) \bmod 20 \text {. }
$$

Definition. Let $\mathscr{G}$ be an additive subgroup of $F$. We say $u$ and $v$ are of the same parity $\bmod \mathscr{G}$ if $Q(\bar{u}) \equiv Q(\bar{v}) \bmod \mathscr{G}$ for all $\bar{u} \in \mathfrak{M}_{u}, \bar{v} \in \mathfrak{M}_{v}$.

We have then the immediate consequence which we mention here only because it is generally slightly easier to apply than Theorem 4.1 itself. 
Proposition 4.6. Let $\operatorname{dim} L \geqq 7$. Then, two maximal vectors $u$ and $v$ having the same lengths are integrally equivalent if and only if (i) $\mathscr{G}\left(\langle u\rangle^{\perp}\right)=\mathscr{G}=\mathscr{G}\left(\langle v\rangle^{\perp}\right)$, and (ii) $u$ and $v$ are of the same parity $\bmod G$.

We wish to make the conjecture here that both Theorem 4.1 and Proposition 4.6 hold for $\operatorname{dim} L=4,5,6$ in the general situation as well.

\section{Ternary case.}

Proposition 5.1. Let $L$ be ternary unimodular. Then, $u \sim v$ if and only if. (i) there exist vectors $x_{u} \in \mathfrak{M}_{u}, x_{v} \in \mathfrak{M}_{v}$ such that $Q\left(x_{u}\right) \equiv Q\left(x_{v}\right) \bmod 2 \mathscr{O}$ when $\delta \in 2 \mathscr{P} ; \quad Q\left(x_{u}\right) \equiv Q\left(x_{v}\right) \bmod 4 \delta^{-1} \mathscr{P}$ when $|2| \leqq|\delta|<1$; (ii) otherwise, $\mathscr{G}\left(\langle u\rangle^{\perp}\right)=\mathscr{G}\left(\langle v\rangle^{\perp}\right)$.

Proof. Necessity is obvious. As for sufficiency, we put $L=$ $L_{u} \perp \mathcal{O} w_{u}=L_{v} \perp \mathcal{O} w_{v}$, where $L_{u}=\mathcal{O} u+\mathcal{O} x_{u} \& L_{v}=\mathcal{O} v+\mathcal{O} x_{v}$. But, $d L_{u} \cong d L_{v}$ by the Local Square Theorem. Therefore, by Witt $\mathrm{Q}\left(w_{u}\right) \cong \mathrm{Q}\left(w_{v}\right)$ and $F L_{u} \cong F L_{v}$.

It is not difficult to see that $L_{u} \cong L_{v}$ so that $u \sim v$ by Theorem 2.1 since $u, v$ are of the same parity over isometric binary components. (These statements are true provided $\delta \notin \mathscr{W}$. But, then if $\delta \in \mathscr{U}$, condition (ii) finishes the proof immediately.)

6. $\operatorname{dim} L=4,5,6$. Let us put

$$
\begin{aligned}
\mathfrak{M}_{x}^{(s)} & =\left\{w \in \mathfrak{M}_{x} \mid \mathscr{N}(\mathcal{O} x+\mathscr{O} w)^{\perp}=2 \mathscr{P}^{-s}\right\} \\
\mathfrak{M}_{x}^{(s, t)} & =\left\{w \in \mathfrak{M}_{x}^{(s)} \mid \mathscr{W}(\mathscr{O} x+\mathscr{O} w)^{\perp}=2 \mathscr{P}^{-t}\right\} .
\end{aligned}
$$

We shall write $\mathscr{N}(L) \cong \mathscr{N}(K) \bmod \mathscr{A}$ (here $\mathscr{A}$ denotes any fractional ideal in $F$ ) to mean that there exist respective norm generators $a_{L} \in Q(L), a_{K} \in Q(K)$ such that $a_{L} \equiv a_{K} \xi^{2} \bmod \mathscr{A}$ for some unit $\xi$.

Proposition 6.1. Let $L$ be quarternary unimodular. Then, $u \sim v$ if and only if conditions (i) and (ii) in Proposition 5.1 hold, and also $\mathscr{N}\left(\mathcal{O} u+\mathscr{O} x_{u}\right)^{\perp} \cong \mathscr{N}\left(\mathcal{O} v+\mathscr{O} x_{v}\right)^{\perp} \bmod 2 \mathscr{O}$.

Proof. Sufficiency follows closely to the proof of last proposition. Denote $L_{u}$ and $L_{v}$ as before. Again, $L_{u} \cong L_{v}$ by direct computation of the norm groups. Condition

$$
\mathscr{N}\left(\mathcal{O} u+\mathcal{O} x_{u}\right)^{\perp} \cong \mathscr{N}\left(\mathcal{O} v+\mathcal{O} x_{v}\right)^{\perp} \bmod 20
$$

together with $93: 17$ of [6] give us 


$$
L_{u}^{\perp} \cong A\left(a_{u},-\alpha a_{u}^{-1}\right), \quad L_{v}^{\perp} \cong A\left(a_{v},-\alpha a_{v}^{-1}\right)
$$

for the suitable norm generators $a_{u}, a_{v}$ of $L_{u}^{\perp}, L_{v}^{\perp}$ respectively. Now, it is easy to see that $\mathscr{W}\left(L_{u}^{\perp}\right)=\mathscr{W}\left(L_{v}^{\perp}\right)=$ (say $\left.\mathscr{W}\right)$. Thus, $a_{u} \cong$ $a_{v} \bmod \mathscr{W}$, so that $\mathscr{G}\left(L_{u}^{\perp}\right)=\mathscr{G}\left(L_{v}^{\perp}\right)$ and $L_{u}^{\perp} \cong L_{v}^{\perp}$ by Witt and O'Meara.

Direct computations again shows that $u, v$ are of the same parity over isometric binary components and so apply Theorem 2.1. Again, the case of $\delta$ being unitary is trivial.

Proposition 6.2. Suppose $\operatorname{dim} L=5$. Then, $u \sim v$ if and only if: (i) there are vectors $x_{u} \in \mathfrak{M}_{u}^{(e, t)}, x_{v} \in \mathfrak{M}_{v}^{(e, t)}$ for some $t \geqq 0$ such that $Q\left(x_{u}\right) \equiv Q\left(x_{v}\right) \bmod 4 \delta^{-1} \mathscr{P}$ when $\delta \notin 2 \mathscr{P}$; and $Q\left(x_{u}\right) \equiv Q\left(x_{v}\right) \bmod 20$ when $\delta \in 2 \mathscr{P}^{\circ}$ (ii) $\mathscr{G}\left(\langle u\rangle^{\perp}\right)=\mathscr{G}\left(\langle v\rangle^{\perp}\right)$ if $\delta \in \mathscr{U}$. (Here, $e$ denotes ord 2.)

Proof. Using the same $L_{u}$ and $L_{v}$, one proves that they are again isometric. So, $F L_{u} \cong F L_{v}$ by Witt. But now, $\operatorname{dim} L_{u}^{\perp}=\operatorname{dim} L_{v}^{\downarrow}=3$ so that $L_{u}^{\perp}$ represents every weight (base) generator; similarly for $L_{v}^{\perp}$. Put $\mathscr{W}\left(L_{u}^{\perp}\right)=\mathscr{W}^{-}\left(L_{v}^{\perp}\right)=b \mathcal{O}$.

If $\mathscr{N}\left(L_{u}^{\perp}\right) \mathscr{V}\left(L_{u}^{\perp}\right) \sim 1$, (i.e. if $t$ is even) then

$$
L_{u}^{\perp} \cong A(0,0) \perp\langle-d\rangle \cong L_{v}^{\perp},
$$

where $d \cong d L_{u}$. Thus, $u \sim v$ by Theorem 2.1.

If $t$ is odd, then $93: 18$ of [6] shows that $L_{u}^{\perp} \cong A(b, 0) \perp\langle-d\rangle$ if $F L_{u}$ is isotropic, and $L_{u}^{\perp} \cong A\left(b, 4 \rho b^{-1}\right) \perp\langle-d(1-4 \rho)\rangle$ if $F L_{u}$ anistropic. Similarly, we write out for $L_{v}^{\frac{1}{}}$. Thus, $L_{u}^{\perp} \cong L_{v}^{\perp}$ always. Apply Theorem 2.1.

Proposition 6.3. Suppose $\operatorname{dim} L=6$. Then, $u \sim v$ if and only if: (i) there are vectors $x_{u} \in \mathfrak{M}_{u}^{(s, t)}, x_{v} \in \mathfrak{M}_{v}^{(s, t)}$ for some $s, t \geqq 0$ such that $Q\left(x_{u}\right) \equiv Q\left(x_{v}\right) \bmod 20$ for $\delta \in 2 \mathscr{P}$; and congruence modulo $4 \delta^{-1} \mathscr{P}$ if $\delta \notin 2 \mathscr{P}$; (ii) $\mathscr{N}\left(\mathcal{O} u+\mathcal{O} x_{u}\right)^{\perp} \cong \mathscr{N}\left(\mathcal{O} v+\mathcal{O} x_{v}\right)^{\perp} \bmod 2 \mathscr{P}^{-t}$; and (iii) $\mathscr{G}\left(\langle u\rangle^{\perp}\right)$ equals $\mathscr{G}\left(\langle v\rangle^{\perp}\right)$ if $\delta \in \mathscr{U}$.

\section{REFERENCES}

1. J. S. Hsia, On Integral Witt's Theorem over Dyadic Local Fields, M. I. T. Ph. D. Thesis 1966.

2. - Integral Equivalence for Vectors over Depleted Modular Lattices on Dyadic Local Fields, to appear in Amer. J. Math.

3. D. G. James, Integral invariants for vectors over local Fields, Pacific J. Math. 15 (1965), 905-916.

4. M. Knebusch, Assoziierte Vektoren in Maximalen Gittern Lokaler Quadratischer Raume, Math. Z. 89 (1965), 213-223. 
5. O. T. O'Meara, Integral equivalence of quadratic forms in ramified local fields, Amer. J. Math. 79 (1957), 157-186.

6. - Introduction to Quadratic Forms, Grundlehren der Mathematischen Wissenschaften, Springer-Verlag, Berlin, 1963.

7. C. R. Riehm, On the integral representations of quadratic forms over local fields, Amer. J. Math. 86 (1964), 25-62.

8. S. Rosenzweig, An Analogy of Witt's Theorem for Modules over the Ring of $p$ adic Integers, M. I. T. Ph. D. Thesis 1958.

9. A. Trojan, The integral extension of isometries of quadratic forms over local fields, Canad. J. Math. 18 (1966), 920-942.

10. C. T. C. Wall, On the orthogonal groups of unimodulor quadratic forms, Math. Ann. 147 (1962), 328-338.

Received January 31, 1967, and in revised form April 2, 1967.

The OHio State University

Columbus, OHio 


\section{PACIFIC JOURNAL OF MATHEMATICS}

\section{H. ROYDEN}

Stanford University

Stanford, California

\author{
J. P. JANS \\ University of Washington \\ Seattle, Washington 98105
}

\section{EDITORS}

\author{
J. DugundJI \\ Department of Mathematics \\ Rice University \\ Houston, Texas 77001 \\ Richard ARENS \\ University of California \\ Los Angeles, California 90024
}

\section{ASSOCIATE EDITORS}
E. F. BECKENBACH
B. H. NeumanN
F. WOLF
K. YOSIDA

\section{SUPPORTING INSTITUTIONS}

\author{
UNIVERSITY OF BRITISH COLUMBIA \\ CALIFORNIA INSTITUTE OF TECHNOLOGY \\ UNIVERSITY OF CALIFORNIA \\ MONTANA STATE UNIVERSITY \\ UNIVERSITY OF NEVADA \\ NEW MEXICO STATE UNIVERSITY \\ OREGON STATE UNIVERSITY \\ UNIVERSITY OF OREGON \\ OSAKA UNIVERSITY \\ UNIVERSITY OF SOUTHERN CALIFORNIA
}

\author{
STANFORD UNIVERSITY \\ UNIVERSITY OF TOKYO \\ UNIVERSITY OF UTAH \\ WASHINGTON STATE UNIVERSITY \\ UNIVERSITY OF WASHINGTON \\ AMERICAN MATHEMATICAL SOCIETY \\ CHEVRON RESEARCH CORPORATION \\ TRW SYSTEMS \\ NAVAL ORDNANCE TEST STATION
}

Mathematical papers intended for publication in the Pacific Journal of Mathematics should be typewritten (double spaced). The first paragraph or two must be capable of being used separately as a synopsis of the entire paper. It should not contain references to the bibliography. Manuscripts may be sent to any one of the four editors. All other communications to the editors should be addressed to the managing editor, Richard Arens at the University of California, Los Angeles, California 90024.

50 reprints per author of each article are furnished free of charge; additional copies may be obtained at cost in multiples of 50 .

The Pacific Journal of Mathematics is published monthly. Effective with Volume 16 the price per volume (3 numbers) is $\$ 8.00$; single issues, $\$ 3.00$. Special price for current issues to individual faculty members of supporting institutions and to individual members of the American Mathematical Society: $\$ 4.00$ per volume; single issues $\$ 1.50$. Back numbers are available.

Subscriptions, orders for back numbers, and changes of address should be sent to Pacific Journal of Mathematics, 103 Highland Boulevard, Berkeley 8, California.

Printed at Kokusai Bunken Insatsusha (International Academic Printing Co., Ltd.), 7-17, Fujimi 2-chome, Chiyoda-ku, Tokyo, Japan.

PUBLISHED BY PACIFIC JOURNAL OF MATHEMATICS, A NON-PROFIT CORPORATION

The Supporting Institutions listed above contribute to the cost of publication of this Journal, but they are not owners or publishers and have no responsibility for its content or policies. 


\section{Pacific Journal of Mathematics}

A. A. Aucoin, Diophantine systems ............................. 419

Charles Ballantine, Products of positive definite matrices. I ............... 427

David Wilmot Barnette, A necessary condition for d-polyhedrality ............ 435

James Clark Beidleman and Tae Kun Seo, Generalized Frattini subgroups of finite groups ......................................... 441

Carlos Jorge Do Rego Borges, A study of multivalued functions ............. 451

William Edwin Clark, Algebras of global dimension one with a finite ideal

lattice ...............................................

Richard Brian Darst, On a theorem of Nikodym with applications to weak convergence and von Neumann algebras .........................

George Wesley Day, Superatomic Boolean algebras .....................

Lawrence Fearnley, Characterization of the continuous images of all

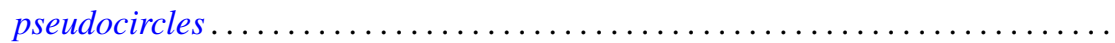

Neil Robert Gray, Unstable points in the hyperspace of connected subsets....... 515

Franklin Haimo, Polynomials in central endomorphisms .................. 521

John Sollion Hsia, Integral equivalence of vectors over local modular lattices . . . . 527

Jim Humphreys, Existence of Levi factors in certain algebraic groups .......... 543

E. Christopher Lance, Automorphisms of postliminal $C^{*}$-algebras ............ 547

Sibe Mardesic, Images of ordered compacta are locally peripherally metric . . . . 557

Albert W. Marshall, David William Walkup and Roger Jean-Baptiste Robert Wets,

Order-preserving functions: Applications to majorization and order

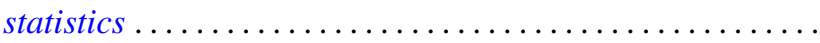

Wellington Ham Ow, An extremal length criterion for the parabolicity of

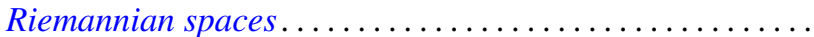

585

Wellington Ham Ow, Criteria for zero capacity of ideal boundary components of

Riemannian spaces...................................... 591

J. H. Reed, Inverse limits of indecomposable continua .................. 597

Joseph Gail Stampfli, Minimal range theorems for operators with thin spectra . . . 601

Roy Westwick, Transformations on tensor spaces..................... 613

Howard Henry Wicke, The regular open continuous images of complete metric

spaces ........................................... 621

Abraham Zaks, A note on semi-primary hereditary rings .............. 627

Thomas William Hungerford, Correction to: "A description of $\operatorname{Mult}_{i}\left(A^{1}, \cdots, A^{n}\right)$

by generators and relations" ............................. 629

Uppuluri V. Ramamohana Rao, Correction to: "On a stronger version of Wallis' formula".............................................. 629

Takesi Isiwata, Correction: "Mappings and spaces" ................... 630

Henry B. Mann, Josephine Mitchell and Lowell Schoenfeld, Correction to:

"Properties of differential forms in $n$ real variables" .... . .

James Calvert, Correction to: "An integral inequality with applications to the

Dirichlet problem"............................. 